\title{
Análisis de riesgos climáticos sobre los componentes, recursos y medios de vida de los sistemas alimentarios del departamento de Copán en el año 2014
}

Juan Manuel López Guevara ${ }^{1}$

\section{RESUMEN}

El estudio analiza los riesgos de los sistemas alimentarios en dos comunidades del departamento de Copán ante los efectos de las amenazas climáticas, mediante la estimación de la peligrosidad de las amenazas identificadas en la zona y del grado de sensibilidad, exposición y capacidad de adaptación ante los impactos climáticos de las amenazas identificadas. La estrategia de diseño de la investigación es un estudio de caso instrumental con unidades incrustadas. La muestra es no probabilística y sigue una estrategia de selección deliberada e intencional de informantes, aplicando la entrevista semiestructurada y los grupos focales como técnicas de recolección de la información. La investigación analiza la peligrosidad de las amenazas climáticas y cómo estas inciden sobre los medios de vida y los recursos de los sistemas alimentarios; asimismo, la sensibilidad de los sistemas alimentarios con respecto a los componentes de la seguridad alimentaria y la exposición de los recursos, servicios, organizaciones y políticas de apoyo de los sistemas alimentarios ante las amenazas climáticas; y la capacidad de adaptación con respecto al acceso, flexibilidad y estabilidad de los recursos y medios de vida. La vulnerabilidad, se determinó como una función de la sensibilidad, exposición y capacidad de adaptación. Por último, se determinó el riesgo de los sistemas alimentarios de las comunidades ante las amenazas climáticas, como una función de las amenazas y la vulnerabilidad.

Palabras claves: riesgos, vulnerabilidad, sistemas alimentarios, amenazas climáticas, medios de vida, seguridad alimentaria

1 Profesor universitario beneficiario de una beca básica de la DICYP, carrera de Ingeniería Agroindustrial, UNAH-CUROC: lopezgjm@gmail.com 


\section{ABSTRACT}

The study assesses the risks that are found in the food systems of two communities in Copán, Honduras, due to the exposure of climate hazards. The danger of climate hazards identified in the zone and the sensitivity was valued, as well as the exposure and adaptive capacity degree due to the climate impacts of the identified hazards. The investigation's design strategy is an instrumental case study with embedded units. The sample is non-probabilistic, following a deliberate and intentional selection of key informants, and the application of a semi-structured interviews and focal groups as information collection techniques. The research analyses (1) the degree of danger in climate hazards, and how they influence over the livelihoods and resources of the food systems; (2) the sensitivity of food systems with regard to the food security dimensions and the exposure of food systems resources, services, organizations and support policies due to climate hazards, and; (3) the adaptive capacity regarding access, flexibility and stability of resources and livelihoods. The vulnerability degree was determined as a function of sensitivity, exposure and adaptive capacity. At last, the risk of communities' food systems due to climate hazards, was determined as a function of hazards and vulnerability.

Key words: risks, vulnerability, food systems, climate hazards, livelihoods, food security. 


\section{INTRODUCCIÓN}

El estudio tiene su origen en la necesidad cada vez más importante de vincular la vulnerabilidad climática y la seguridad alimentaria, a través de enfoques que comprendan el análisis de los medios de vida de las comunidades ante los cambios que el clima provoca en sus entornos. Tomando en consideración que los impactos negativos de la variabilidad y el cambio climático en la seguridad alimentaria han sido ampliamente reconocidos, no obstante, la mayor parte de la investigación se ha centrado, hasta la actualidad, en los efectos climáticos directos en la productividad de los cultivos (Schmidhuber y Tubiello, 2007).

Aspectos importantes de la vulnerabilidad en la inseguridad alimentaria, como la estabilidad de acceso y utilización de los alimentos, así como las variaciones en la vulnerabilidad a través de escalas espaciales y temporales, son menos conocidos. Por tal razón, el entendimiento de los impactos del cambio climático sobre los componentes de la seguridad alimentaria y de los nexos entre la seguridad alimentaria y el cambio climático a nivel local, requiere, además del conocimiento sobre las condiciones climáticas, sus tendencias y la modelación de escenarios futuros del comportamiento de los cultivos, comprensión de los medios de vida y de los recursos de las comunidades y de las organizaciones y políticas relacionadas con los sistemas alimentarios.

Es, por lo tanto, esencialmente en el análisis de los riesgos climáticos sobre los sistemas alimentarios a nivel local o de microcuencas, incluir los diferentes componentes de la seguridad alimentaria, los recursos y servicios de apoyo de los sistemas alimentarios como la tierra, el agua, los bosques, los caminos y otro tipo de infraestructura productiva y el marco político y económico constituido por las organizaciones comunitarias, municipales y regionales y las políticas de apoyo de los sistemas alimentarios.

En la región occidental de Honduras no se conocen antecedentes de utilización de herramientas que proporcionen información sobre las tendencias del cambio climático y la seguridad alimentaria a nivel local, que ayuden a evaluar los nexos del cambio climático con los componentes de la seguridad alimentaria, los recursos locales y el acceso a los medios de vida. En consecuencia, la utilización de esta herramienta para la evaluación de la vulnerabilidad y riesgos de los sistemas alimentarios ante el cambio climático a nivel de las comunidades o microcuencas, pretende contribuir a llenar los vacíos de conocimiento en el análisis sobre el tema. 
La información podrá servir para que las comunidades mejoren su capacidad local de adaptación, de manera que puedan hacer ajustes, moderar o aprovechar los cambios que el clima provoca en sus entornos. Se pretende que los resultados sean de utilidad también para las organizaciones que intervienen en las comunidades, cuya información puede servir en la formulación de propuestas de proyectos relacionados con el tema; igualmente, para los tomadores de decisiones y profesionales, quienes necesitan comprender y tener las herramientas necesarias para medir los efectos del cambio climático sobre los sistemas alimentarios.

Como resultado de esta investigación, se espera proponer medidas de adaptación de los sistemas alimentarios de las comunidades en el contexto del cambio climático, que puedan ser utilizadas por las instituciones que intervienen en las comunidades y por estas mismas, desarrollando indicadores para medir los cambios en el tiempo, estableciendo mecanismos de coordinación para el seguimiento de los indicadores identificados.

Se utilizó una herramienta metodológica adaptada a las características socioeconómicas, biofísicas y culturales de las comunidades y del entorno de la región occidental del país, que podrá ser utilizada para aplicarse en ambientes y condiciones similares para la evaluación de los riesgos de los sistemas alimentarios de las comunidades ante las amenazas climáticas y su capacidad de adaptación. CRISTAL Seguridad Alimentaria, es una herramienta para la identificación comunitaria de riesgos, adaptación y medios de vida, adaptada por el Instituto Internacional para el Desarrollo Sostenible [IISD] (2014), en forma conjunta con otras instituciones. A partir de esta herramienta se aprovecha el modelo de evaluación de impacto ambiental (EIA) y el marco para medios de vida sostenibles (SLF, por sus siglas en inglés), que proponen un proceso lógico y de fácil aplicación para ayudar a quienes lo utilicen a entender mejor los nexos entre los riesgos relacionados con el clima y los medios de vida de las personas (IISD, Intercooperation, SEI, 2009).

\section{DISEÑO}

La investigación presenta un enfoque cualitativo, ya que se evalúan categorías cualitativas en los procesos de recolección, análisis e interpretación de la información. La estrategia de diseño es un estudio de caso, en el cual se analiza la realidad con respecto a la vulnerabilidad alimentaria de las comunidades ante los efectos del cambio climático, utilizando métodos hermenéuticos (interpretativos), tratando de 
observar los fenómenos de estudio y buscarles significado.

Ya que no se hace ninguna manipulación de las variables, sino que solamente se observan los fenómenos relacionados con los efectos del cambio climático en los sistemas alimentarios de las comunidades, tal como se presentan en el contexto natural, para posteriormente analizarlos, la investigación es no experimental. Asimismo, se puede clasificar, de acuerdo a Hernández, Fernández y Baptista (2010), como un estudio de caso instrumental, ya que provee de insumos de conocimiento acerca del tema de los riesgos de los sistemas alimentarios ante el cambio climático, además porque podrá servir para aplicar otros casos similares de análisis. Puesto que los datos para la realización de los análisis se recolectaron en un solo momento, en un período de tiempo determinado, la investigación se caracteriza bajo un alcance transversal o transeccional.

Población sobre la que se ha hecho el estudio

El estudio se desarrolló basándose en el análisis de dos casos correspondientes a las comunidades de Vega Redonda y Buena Vista, evaluando a cada uno de forma integral. La selección de las comunidades se realizó tomando en consideración el conocimiento previo de la diversidad de medios de vida y la ocurrencia de amenazas climáticas representativas. Para cada caso se hizo el proceso de recolección de datos y análisis de manera individual, sin perder de vista el caso completo.

En este contexto, se puede considerar como un estudio de caso con unidades incrustadas, ya que las unidades de análisis dentro del caso corresponden a las unidades familiares de las comunidades y a las mismas comunidades estudiadas. Esto se justifica ya que se analizaron aspectos relacionados con la utilización, acceso y disponibilidad de los alimentos, relacionados directamente con el núcleo familiar; pero, también se estudiaron los recursos y medios de vida de las comunidades y las amenazas climáticas que inciden sobre las mismas.

La muestra obtenida es no probabilística, ya que no busca generalizar resultados en una población. Se propuso una estrategia de selección deliberada e intencional de informantes. La identificación y caracterización de los individuos se hizo de acuerdo con el grado de conocimientos acerca del entorno de las comunidades, los sistemas productivos y las amenazas climáticas que han afectado a las mismas en las últimas décadas. Se determinó como informantes a líderes comunitarios, miembros de organizaciones comunitarias y personas con conocimientos de la historia de las comunidades y del contexto de las mismas. 


\section{Entorno}

El estudio se realizó en las comunidades de Buena Vista, del municipio de San José, y Vega Redonda, del municipio de Dolores; ambas en el departamento de Copán, en la región occidental de la República de Honduras. Dichas comunidades forman parte de la mancomunidad de municipios Higuito. Se encuentran aproximadamente a la misma distancia de la cabecera departamental (Santa Rosa de Copán) y presentan características biofísicas similares: temperaturas entre 20 y $30{ }^{\circ} \mathrm{C}$, precipitaciones entre 1300 y $1600 \mathrm{~mm}$ de agua por año, con aproximadamente unos $200 \mathrm{~m}$ de diferencias de altitud: $700 \mathrm{msnm}$ en Vega Redonda y $950 \mathrm{msnm}$ en Buena Vista (Consejo Intermunicipal Higuito, 2006). Las actividades agrícolas son el medio de vida de mayor importancia en las comunidades, cuyos habitantes se dedican al cultivo de maíz, frijol, café, ganado vacuno y algunos cultivos hortícolas en muy pequeña escala.

\section{Intervenciones}

El método de análisis considera un diseño sistemático, ya que se enfoca en el "empleo de ciertos pasos en el análisis de datos" (Hernández, Fernández y Baptista, 2010, p. 493), cuya codificación es axial, puesto que se agrupan los datos "para crear conexiones entre categorías y temas" (Hernández, Fernández y Baptista, 2010, p. 494). El diseño ayuda a comprender el fenómeno de los riesgos climáticos sobre los sistemas alimentarios, ya que identifica a los conceptos implicados y la secuencia de acciones e interacciones de los participantes involucrados.

La herramienta adaptada para la recolección y análisis de la información proporciona un marco para organizar, en un formato lógico, la información reunida a nivel local (de las comunidades y los informantes claves). Se elaboró tomando como base la herramienta CRISTAL Seguridad Alimentaria (IISD, 2014), a partir de las categorías de análisis: perturbaciones y amenazas climáticas y sistemas alimentarios.

Los datos se obtuvieron utilizando técnicas de recolección de la información como la observación, aplicando lineamientos para observación diseñados previamente con los elementos de interés de acuerdo a las variables del estudio; entrevistas semiestructuradas, dirigidas a informantes claves de las comunidades y desarrollo de grupos focales en talleres de trabajo con la participación de líderes comunitarios y otros informantes claves. 


\section{ANÁLISIS DE LOS DATOS}

El proceso general de análisis de datos consistió en la reducción (categorización y codificación de elementos), la transformación y disposición de los datos (aplicación de funciones lógicas de análisis de datos), la obtención de resúmenes y la obtención y verificación de conclusiones. La tabulación y análisis de datos se realizó utilizando páginas de Excel, en las que se incluyeron hojas de cálculo siguiendo una secuencia de análisis de acuerdo a las variables de investigación. El estudio describe y analiza los siguientes elementos:

1. Los principales medios de vida y fuentes de ingreso de las comunidades y las principales amenazas climáticas, determinando un índice de significancia en función de la frecuencia y la intensidad de la amenaza.

2. Los impactos de las amenazas climáticas sobre tres componentes de la seguridad alimentaria (utilización, acceso y disponibilidad de los alimentos), determinando un índice de sensibilidad climática para cada uno, a partir de la importancia de los alimentos y los impactos de las amenazas climáticas.

3. Los impactos sobre los recursos, servicios, organizaciones y políticas de apoyo de los sistemas alimentarios, obteniendo índices de exposición ante las amenazas climáticas.

4. La capacidad de adaptación que se fundamenta en la identificación de estrategias de adaptación para los diferentes recursos y medios de vida de las comunidades, definidos en forma de atributos como el acceso, la flexibilidad y la estabilidad, para los cuales se identifican indicadores para los que se determinaron índices de capacidad de adaptación.

El índice de vulnerabilidad de los sistemas alimentarios de las comunidades ante las amenazas climáticas se obtuvo como una función de la sensibilidad, exposición y capacidad de adaptación (Brooks, 2003; Johnston y Williamson, 2007). Por último, se definió el riesgo como una función del índice de significancia de las amenazas y del índice de vulnerabilidad.

Los índices se desarrollaron de acuerdo a parámetros estandarizados, utilizando funciones de Microsoft Excel, de tal manera que se estableciesen categorías con los mismos criterios en cada uno de los diferentes aspectos de análisis, para generar 
escalas de alores que al ir vinculándose originen los índices de vulnerabilidad y riesgo.

\section{RESULTADOS}

Los sistemas alimentarios locales y los cambios climáticos y amenazas climáticas observadas en el entorno

Las principales actividades de medios de vida que constituyen fuentes de ingresos en las comunidades son la agricultura, el trabajo por jornal y la ganadería. En Buena Vista se puede añadir la industria de la madera y en Vega Redonda, las remesas. En las actividades agrícolas predomina la producción de maíz y frijol (ambos, principalmente para el autoconsumo) y café. Otros rubros se explotan en pequeña escala, como las hortalizas, pimienta gorda, banano criollo y plátano.

Los cambios climáticos observados en el entorno de las comunidades son el incremento de las temperaturas, la irregularidad de las precipitaciones (mayor presencia de lluvias en los meses de verano, de manera errática; por otra parte, inviernos más cortos y menos intensos), mayor ocurrencia de eventos extremos y la alteración del ciclo de los seres vivos en los ecosistemas.

Las amenazas climáticas comunes identificadas en las comunidades son los movimientos de laderas y la irregularidad en el régimen de las precipitaciones. En la comunidad de Buena Vista se incluyen, además, los vientos fuertes y las sequías y en Vega Redonda, las inundaciones y la erosión del suelo. En general, el índice promedio de significancia de las amenazas en las comunidades es de baja a media. Particularmente, en la comunidad de Buena Vista hay una amenaza de media a alta de los vientos fuertes; no obstante, las amenazas relacionadas con los cambios que se presentan con el régimen de las lluvias al alterar el desarrollo de las actividades agropecuarias - que son el principal medio de vida de la comunidad-, con la sequía y sus efectos en la disponibilidad de agua para uso doméstico, son las principales preocupaciones de la comunidad.

En la comunidad de Vega Redonda, los resultados obtenidos indican que hay una significancia media de cada una de las amenazas. A pesar de que no se registran amenazas significativas, es importante tomar en cuenta los fenómenos hidrometeorológicos que pueden causar inestabilidad de los suelos, erosión e 
inundaciones. Una preocupación importante en la comunidad son las perturbaciones generadas por los cambios en el régimen de las precipitaciones, ya que provoca alteraciones en las actividades productivas agropecuarias, que son los principales medios de vida de la comunidad.

\section{Análisis de vulnerabilidad de las comunidades ante las amenazas climáticas}

El análisis de vulnerabilidad se realiza determinando el grado de sensibilidad, exposición y capacidad de adaptación de los sistemas alimentarios ante las amenazas climáticas:

1. Sensibilidad de los componentes de los sistemas alimentarios ante las amenazas naturales: en Buena Vista, se presenta una sensibilidad media de los componentes de la seguridad alimentaria de la comunidad ante el impacto de las amenazas climáticas. El acceso a los alimentos se estima como el componente más sensible. Los impactos de los fenómenos climáticos que inciden sobre la producción de alimentos se muestran como los más importantes. En la comunidad de Vega Redonda, en general, se presenta una sensibilidad media. La utilización y la disponibilidad de los alimentos se valoran como los componentes más sensibles. Los impactos de los fenómenos climáticos sobre aspectos de la utilización de los alimentos como el agua y saneamiento y sus efectos sobre la salud humana, así como sobre los recursos claves de la disponibilidad alimentaria, como la producción, almacenamiento, transporte y ventas de los alimentos, se muestran como los más importantes.

En ambas comunidades, los cambios en el régimen de las lluvias son las perturbaciones climáticas que más preocupan por su incidencia sobre la producción agropecuaria y, por consiguiente, sobre los componentes de la seguridad alimentaria. Estos cambios afectan la producción de alimentos, incrementando la incidencia y frecuencia de plagas y enfermedades en los cultivos, alterando los ciclos reproductivos de las especies y la programación de las actividades agrícolas.

2. Exposición de los recursos y servicios de apoyo ante las amenazas climáticas: el índice de exposición de los recursos y servicios de apoyo ante las amenazas climáticas en la comunidad de Buena Vista, muestra que los recursos naturales y financieros presentan una exposición de baja a media, mientras que los recursos físicos presentan de ninguna a baja exposición. En la comunidad de Vega Redonda, el índice de exposición muestra que los recursos de la 
comunidad presentan una exposición de baja a media, aunque individualmente algunos presentan mayor y otros menor exposición.

La mayor exposición de los recursos de las comunidades se presenta ante la irregularidad en el régimen de las lluvias. Los recursos naturales son los más expuestos, siendo el recurso tierra afectado ante problemas erosivos, inestabilidad de laderas y al ser perturbadas las condiciones de vida de las especies vegetales y modificados los hábitats de plagas y enfermedades.

Específicamente en Buena Vista, la exposición ante la sequía también es muy importante en todos los recursos de la comunidad, siendo más crítica en los recursos naturales por sus efectos en el agua, el suelo y el bosque. Las limitaciones actuales de acceso y disponibilidad de agua en la comunidad se constituyen en factores agravantes ante la sequía, en términos de menores precipitaciones totales o extensión del período seco, llevando consecuentemente a una menor humedad del suelo y reducción de la disponibilidad de agua del acuífero.

En Vega Redonda, la exposición del recurso suelo ante la erosión hídrica constituye una perturbación cuyos efectos se manifiestan sobre la agricultura y la seguridad alimentaria en la comunidad. El grado de exposición, a su vez, está vinculado con la cobertura vegetal, la pendiente de los terrenos y la pluviometría. El efecto de la erosión es particularmente relevante en los suelos de laderas y en aquellos cuyos sistemas de producción son inapropiados, lo que se traduce en la pérdida de la fertilidad de los suelos y en los rendimientos en las actividades agrícolas.

3. Exposición de las organizaciones y políticas de apoyo ante las amenazas climáticas: en la comunidad de Buena Vista, el índice de exposición de las organizaciones y políticas de apoyo ante las amenazas naturales muestra que los recursos presentan una exposición de ninguna a baja, deduciendo que tanto las organizaciones comunitarias, la toma de decisiones y las costumbres en la comunidad no son afectadas (o lo son levemente) por la ocurrencia de las amenazas naturales. Por su parte, en la comunidad de Vega Redonda, el índice de exposición muestra que los recursos sociales y políticos presentan una exposición de baja a media, mientras que los recursos culturales muestran una menor exposición ante las amenazas climáticas. La exposición de los recursos sociales y políticos se explica por el hecho de que algunas amenazas climáticas, como las inundaciones y los movimientos de laderas, pueden afectar los procesos de gestión y las 
acciones que las organizaciones comunitarias realicen, en los que se requiera movilización fuera de la comunidad.

4. Exposición de las comunidades ante las amenazas naturales: en resumen, de acuerdo a los índices obtenidos, se concluye que en ambas comunidades los recursos y servicios de apoyo presentan un grado de exposición de baja a media, mientras que las organizaciones y políticas de apoyo a los sistemas alimentarios presentan de ninguna a baja exposición ante las amenazas climáticas.

5. Capacidad de adaptación de las comunidades: en general, en ambas comunidades, los índices de la capacidad de adaptación muestran un regular acceso, flexibilidad y estabilidad. El índice muestra una capacidad de adaptación de regular a deficiente de las comunidades, como también se muestran los índices de las estrategias para casi todos los recursos comunitarios.

Análisis de riesgo de las comunidades ante las amenazas climáticas

El análisis de riesgo de los sistemas alimentarios ante las amenazas climáticas intenta determinar una magnitud de las consecuencias adversas para los sistemas alimentarios, después de eventos climáticos de peligro. De manera que esta magnitud es una función de la interacción entre las posibles amenazas de la zona y la vulnerabilidad de los sistemas alimentarios.

El índice de riesgo en Buena Vista y de Vega Redonda, muestra que las comunidades presentan un riesgo alto de daño de los sistemas alimentarios ante la ocurrencia de las amenazas identificadas. Los diferentes componentes de los sistemas alimentarios (utilización, acceso, disponibilidad), así como los recursos y servicios de apoyo de los sistemas alimentarios, presentan probabilidades de sufrir daños en una magnitud que puede ser alta, ante la posibilidad de ocurrencia de contingencias climáticas.

De igual manera, algunas amenazas y perturbaciones climáticas varían entre las comunidades y en los diferentes aspectos de la vulnerabilidad; no obstante, en general, el grado de daños y pérdidas podría tener similar magnitud en ambas comunidades en los elementos que son expuestos ante las amenazas.

De manera general, se presentan algunos aspectos comunes del riesgo de las comunidades ante las amenazas climáticas, siguiendo la línea de análisis desde los componentes de la seguridad alimentaria hasta los recursos y servicios de apoyo de 
los sistemas alimentarios:

1. Con respecto a la utilización de los alimentos, los riesgos se sitúan en los aspectos relacionados con el estado de salud de las personas y su capacidad para absorber nutrientes; problemas relacionados con el acceso al agua y a los servicios de saneamiento y a la ocurrencia de enfermedades vinculadas con las condiciones de salubridad de la población; además, con la baja capacidad de las comunidades de preparar y almacenar alimentos en sus hogares que puedan proveer suministros para tiempos de crisis.

2. Acerca del acceso a los alimentos, los riesgos se encuentran en función de las estrategias. Si los hogares cuentan con diversas maneras de tener alimentos durante el año, se reducen los riesgos de carencia de estos. Se puede observar que las estrategias de acceso en las comunidades se centran en una o dos con respecto a cada alimento, lo que hace más difícil llegar al mismo en tiempo de crisis. Otro riesgo está relacionado con la inexistencia de sistemas de almacenamiento en las comunidades a nivel colectivo o comunitario y con la deficiencia de los sistemas familiares, lo que impide que puedan mantener excedentes en tiempos de crisis. Los riesgos de la producción de alimentos se elevan ante la carencia de tecnologías que puedan hacer frente a las amenazas climáticas (riego, semillas mejoradas, invernaderos), ante la distribución inequitativa de la tierra y la inexistencia de apoyo para la producción (financiero y técnico), entre otros factores.

3. En cuanto a los recursos y servicios de apoyo de los sistemas alimentarios, los riesgos subyacentes ante la posibilidad de ocurrencia de las amenazas climáticas se orientan al deterioro de los recursos (por ejemplo, del suelo por la erosión, que es una situación de suma preocupación en las comunidades en donde se visualiza la progresiva degradación del recurso ante la pérdida de la capa superficial de uso agrícola), a la disminución de los recursos (por ejemplo, la preocupación de las comunidades por la disminución del recurso agua que se ha visto en los últimos años o por la reducción de las áreas de bosques de las comunidades), a posibles interrupciones de los servicios que prestan algunos recursos (caminos, suministro de agua, energía eléctrica) o a la posibilidad de que se propague rápidamente un fallo en los servicios de infraestructura vinculados al recurso o servicio si se produce un descuido en uno de los elementos, como la ocurrencia de daños en algún componente del sistema de agua que pueden dañar otros componentes y causar el colapso del sistema. 
4. Las organizaciones y políticas de apoyo de los sistemas alimentarios de las comunidades expresan riesgo ante su virtual incapacidad para restablecer el funcionamiento después de una perturbación o estrés climático, ante la falta de autonomía, dependencia de otras instancias y poca capacidad de ejecutar planes y movilizar recursos.

\section{DISCUSIÓN}

Un aspecto importante del estudio es la utilización de una herramienta para medir los riesgos climáticos basada en las observaciones de las comunidades, pero centrada en los medios de vida y recursos comunitarios. La herramienta se puede aplicar en las comunidades utilizando técnicas de visualización y dinámica de grupos, de una manera participativa, y puede ser complementada con otras herramientas de apoyo en el levantamiento de información, como las desarrolladas por Geilfus (1997), Wespi, et al. (2001) y CARE (2010).

La tabulación y análisis de la información es fácil y los criterios de categorización pueden ser adaptados o ajustados por los usuarios. No obstante, la aplicación de la herramienta requiere cierto grado de inmersión en la vida de las comunidades para un mejor análisis e interpretación de la información.

En el estudio se desarrollan índices para mostrar la significancia de los fenómenos, tales como semáforos que brindan señales de colores para regular la circulación, indicar claramente qué decisión tomar, qué hacer. Así como cada color es un símbolo que ayuda a distinguir una situación y que puede indicar las acciones a tomar, en el estudio cada categoría obtenida muestra señales para la toma de decisiones, pero que presentan todo un rango interpretativo que requieren ser analizadas dentro de todo el contexto y bajo el conocimiento de aspectos intrínsecos de las condiciones de los fenómenos para obtener interpretaciones realistas.

El estudio provee insumos conceptuales y establece un marco de análisis fundamentado en los conceptos sobre seguridad alimentaria establecidos por la Organización de las Naciones Unidas para la Agricultura y la Alimentación (2006) sobre vulnerabilidad y riesgos climáticos analizados por el Panel Intergubernamental de Cambio Climático (IPCC, 2007), el Programa de las Naciones Unidas para el Desarrollo (UNDP, 2010) y sobre el enfoque de los medios de vida sostenibles 
desarrollado por el Departamento para el Desarrollo Internacional (DFID) del Reino Unido (2001).

Bajo estos principios, se complementa el estudio de amenazas con las metodologías desarrolladas para análisis de inestabilidad de laderas y para inundaciones fluviales por el Instituto Nicaragüense de Estudios Territoriales (INETER, 2005) y para análisis de erosión hídrica desarrollado por el Programa para la Agricultura Sostenible en Laderas de América Central (PASOLAC) y el Centro Internacional de Agricultura Tropical (CIAT, 2005). Sin embargo, la utilización de estas últimas metodologías en la investigación es solamente orientativa para el desarrollo de criterios de categorización de las amenazas. La aplicación rigurosa de estas para cada una de las amenazas implicaría la ejecución de otros procesos investigativos.

También el estudio ubica y analiza el contexto local, haciendo partícipes a los actores locales del análisis de su propia problemática y toma en consideración los recursos y medios de vida de las comunidades, tratando de identificar los nexos entre los riesgos relacionados con el clima y los medios de vida de las personas.

Esta investigación contribuye a facilitar la toma de decisiones. Los responsables locales de la toma de decisiones y las instituciones de apoyo, pueden contar con una herramienta con la que se puedan evaluar de manera sistemática las amenazas que representan la variabilidad y el cambio climático para los principales factores que apuntalan la seguridad alimentaria. En tal sentido, el estudio presenta categorías que brindan valores sobre los fenómenos estudiados, basados en la percepción colectiva de las personas de las comunidades, reduciendo la validez de los juicios a los sujetos que los emiten.

La aplicación de criterios objetivos de análisis, por ejemplo, con respecto a las amenazas climáticas, podría brindar otra perspectiva o complementar los análisis de los estudios. De manera que las acciones propuestas representan solamente algunos ejemplos de posibles medidas de reducción del riesgo que, a la vez, afianzan mejores condiciones de coexistencia con el medioambiente y de satisfacción de necesidades básicas. En consecuencia, no constituyen recetas porque existen particularidades y prioridades que al final determinan las opciones y posibilidades de intervención. 


\section{CONCLUSIONES}

Los cambios climáticos desde la perspectiva de las comunidades, plantean elementos importantes a partir de la observación y conocimientos locales, cuyas consideraciones deberían ser fortalecidas con la introducción de mecanismos para la observación local de una manera más sistemática (observatorios climáticos comunitarios) y complementadas con la información generada por instituciones de investigación.

En general, las amenazas identificadas inciden sobre los medios de vida de las comunidades y sobre los recursos claves de los sistemas alimentarios. La ocurrencia de las amenazas, aunque no se manifieste en situaciones catastróficas inmediatas, puede provocar la alteración de recursos claves para la seguridad alimentaria de las comunidades, dañar sistemas y estructuras comunitarias de apoyo de los sistemas alimentarios y provocar la pérdida o daños de los medios de vida.

La sensibilidad en la utilización de los alimentos muestra alimentos cuyo consumo sufre variación con respecto a la época del año. La variación estacional generalmente presenta descensos en el consumo en el tiempo antes de obtenerse la siguiente cosecha. La pérdida de costumbres alimenticias ancestrales benéficas del consumo de alimentos autóctonos y la precariedad de las condiciones de acceso al agua y saneamiento en las comunidades, son elementos importantes en la determinación del grado de sensibilidad.

Las comunidades combinan diferentes estrategias de acceso a los alimentos. El desarrollo de más de una estrategia de acceso es esencial para la seguridad alimentaria de las comunidades, al ayudarles a afrontar crisis, carencias o dificultades ante las cuales algunos alimentos presenten mayor sensibilidad. La carencia de fuentes de empleo, las características de las organizaciones productivas locales, la falta de apoyo microempresarial, entre otros factores, impiden crear dinámicas en las comunidades en las cuales las familias puedan tener estrategias efectivas de acceso a los alimentos.

Los recursos de la cadena alimentaria manifiestan diferentes grados de sensibilidad ante las amenazas climáticas. Los recursos para la producción de alimentos son los que muestran mayor sensibilidad a los impactos de fenómenos climáticos como las sequías, el exceso de precipitaciones y fenómenos secundarios, como la erosión de 
los suelos. El fortalecimiento de los recursos para la producción, almacenamiento y comercialización de alimentos, a través de acciones concretas de transferencia de tecnología, debería ir también acompañado de cambios estructurales como el mejoramiento de la tenencia de la tierra, acciones de capacitación, acceso al financiamiento, asesoría y acompañamiento en aspectos organizativos, productivos, de mercado, etc.

Los recursos, servicios, organizaciones y políticas de apoyo de los sistemas alimentarios constituyen el tejido que ayuda a cohesionar los componentes de la seguridad alimentaria y a explicar situaciones como la capacidad de respuesta, de administrar y movilizar recursos, de manejar emergencias, etc. En general, se muestra una capacidad de adaptación de regular a deficiente en las comunidades estudiadas, explicada por debilidades, limitaciones y carencias en las estrategias de acceso, flexibilidad y estabilidad de los recursos y medios de vida; lo cual es evidente en la inexistencia de mecanismos y estructuras de prevención y respuesta local y en las debilidades de las organizaciones locales.

\section{AGRADECIMIENTOS}

A los colegas Alfonso Carranza y Kevin Mejía, por su participación en la obtención de datos preliminares que sirvieron de base para la investigación. Al coordinador regional de Investigación Científica del CUROC, Kevin Estévez, por todo el apoyo en la ejecución de la investigación. A la Dirección de Investigación Científica de la UNAH, por el apoyo para el desarrollo del estudio a través de una beca de investigación y la asesoría brindada.

\section{REFERENCIAS}

Brooks, N. (2003). Vulnerability, risk and adaptation: a conceptual framework. Norwich: Tindall Centre for Climate Change Research.

CARE International. (2010). Manual para el análisis de capacidad y vulnerabilidad climática. Perú: CARE.

Consejo Intermunicipal Higuito. (2006). Indicadores socioeconómicos línea de base del municipio de Dolores, Copán. Copán: JICA.

Consejo Intermunicipal Higuito. (2006). Indicadores socioeconómicos línea de base 
del municipio de San José de Copán. Copán: JICA.

Departamento para el Desarrollo Internacional. (2001). Guías sobre medios de vida sostenibles. Londres: DFID.

Food and Agriculture Organization. (2006). Informe de políticas. Roma: FAO.

Geilfus, F. (1997). 80 herramientas para el desarrollo participativo: diagnóstico, planificación, monitoreo, evaluación. San Salvador: PROCHALATE-IICA.

Geilfus, F. (2009). Estrategias campesinas, marco de análisis para el desarrollo rural.

Tegucigalpa: IICA.

Hernández, R.; Fernández, C. y Baptista, M. (2010). Metodología de la investigación. México: McGraw Hill Interamericana.

Instituto Nicaragüense de Estudios Territoriales. (2005). Inestabilidad de laderas, mapas de amenazas. Managua: COSUDE.

Intergovernmental Panel on Climate Change. (2007). Cambio climático 2007: informe de síntesis. Suiza: IPCC.

International Institute for Sustainable Development. (2009). CRISTAL. Herramienta para la identificación comunitaria de riesgos-adaptación y medios de vida. Suiza: IISD, Intercooperation, SEI, IUCN.

International Institute for Sustainable Development. (2014). CRiSTAL Food Security 2.0. User's manual. Wiinnipeg: IISD.

Johnston, M. y Williamson, T. (2007). A framework for assessing climate change vulnerability of the Canadian forest sector. The Forest Chronicle, 3, 358-361.

Programa para la Agricultura Sostenible de América Central y Centro Internacional de Agricultura Tropical. (2005). Manual de métodos sencillos para estimar erosión hídrica. Managua: PASOLAC y CIAT.

Schmidhuber, J. y Tubiello, F. (2007). Global food security under climate change. Proceedings of the National Academy of Sciences, 50, 19703-19708.

United Nations Development Programme. (2010). Mapping climate change vulnerability and impact scenarios. New York: UNDP.

Wespi, M.; Ulloa, S.; Weber, G.; Rodríguez, R.; Fischler, M. y Cisnero, J. (2001). Diagnóstico rural participativo. Managua: PASOLAC. 